\title{
Observaciones acerca de los dispositivos móviles
}

\author{
Observations about mobile devices
}

\section{Observações sobre dispositivos móveis}

\author{
Ritha M. Cedeño-Luna ${ }^{\mathrm{I}}$ \\ rithalce@hotmail.com \\ Kleber H. Alcívar-Vaca II \\ kleberalce@hotmail.com \\ Diego A. Ponce-Vásquez ${ }^{\text {III }}$ \\ diego.ponce@ucuenca.edu.ec
}

Recibido: 30 de enero de 2017 * Corregido: 20 de febrero de 2017 * Aceptado: 20 junio de 2017

\footnotetext{
${ }^{\text {I }}$ Programa de Revalidación de la Maestría de Gestión Estratégica de Tecnologías de la Información, Facultad de Ingeniería; Universidad de Cuenca, Campus Central, Cuenca, Ecuador.

II Programa de Revalidación de la Maestría de Gestión Estratégica de Tecnologías de la Información, Facultad de Ingeniería; Universidad de Cuenca, Campus Central, Cuenca, Ecuador.

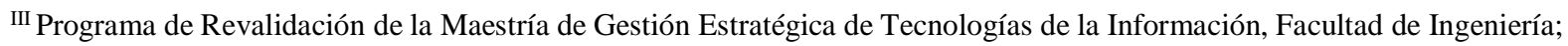
Universidad de Cuenca, Campus Central, Cuenca, Ecuador.
} 


\section{Resumen}

Los dispositivos móviles, como los smartphones (teléfonos inteligentes) o las tablets, son los principales impulsores del cambio en la manera de relacionarse con personas y organizaciones y están abriendo un sinfín de oportunidades en los diferentes sectores de la sociedad. Se realizó una revisión bibliográfica acerca de los dispositivos móviles: antecedentes históricos, funcionalidad e importancia de los dispositivos móviles, tendencia actual de los dispositivos móviles en la educación y su aplicación en el sector empresarial.

Palabras clave: Dispositivos móviles; sector educación; sector empresarial. 


\begin{abstract}
Mobile devices, such as smartphones or tablets, are the main drivers of change in relationships with people and organizations and are opening up endless opportunities in different sectors of society. A literature review was carried out on mobile devices: historical background, functionality and importance of mobile devices, current trends of mobile devices in education and their application in the business sector.
\end{abstract}

Key words: Mobile devices; education sector; business sector. 


\section{Introducción.}

Las telecomunicaciones (del prefijo griego tele, que significa "distancia" o "lejos", o sea "comunicación a distancia") consiste en las técnicas, aparatos, y conocimientos que se utilizan para transmitir un mensaje desde un punto a otro. La página electrónica de la Comisión Federal de Telecomunicaciones define más precisamente a las telecomunicaciones como "toda transmisión, emisión o recepción de signos, señales, escritos, imágenes, sonidos o informaciones de cualquier naturaleza por cable, radioelectricidad, medios ópticos u otros sistemas electromagnéticos." (Rodríguez Luis F. 2012)

Es en esta última definición que aparece la palabra clave en el concepto moderno de las telecomunicaciones: se hacen mediante sistemas electromagnéticos. O sea, que si mando un mensaje mediante una paloma mensajera o le grito a mi vecino desde mi casa, estos dos ejemplos ya no contarían modernamente como telecomunicaciones. Curiosamente, la transmisión mediante señales de humo o mediante espejos, si contarían como telecomunicaciones porque estaríamos usando a la luz (que es un medio electromagnético) para transmitir la información. El número de actividades que cae dentro del rubro de las telecomunicaciones es cada vez más grande: tanto el teléfono común como el celular, las estaciones de radio y televisión, el Internet, la radioastronomía, etc. (Rodríguez Luis F. 2012)

Antecedentes históricos de los dispositivos móviles

El móvil se inicia a los principios de la Segunda Guerra Mundial, donde ya se veía que era necesaria la comunicación a distancia, es por eso que la compañía Motorola creó un equipo llamado 
Handie Talkie H12-16, que es un equipo que permite el contacto con las tropas vía ondas de radio que en ese tiempo no superaban más de 600 Khz. (Historia del móvil .2013)

Los dispositivos móviles se han vuelto uno de los mejores inventos que han existido. La primera generación de dispositivos móviles que se crearon utilizaba múltiples sitios conectados, y se podían recibir llamadas de un sitio a otro. La primera red celular fue hecha en el año 1977 en Chicago y comenzó a funcionar bien en 1978. Después de este año contaba con un aproximado de 1300 clientes. En 1979 una red celular fue lanzada en Japón por NTT. Esta red cubría toda el área de Tokyo, con 23 estaciones base a las que se comunicaba. Después ésta red se expandió hasta cubrir todo Japón y se convirtió en la primera red 1 G nacional. (Cárdena G 2012)

En un principio éstos dispositivos sólo funcionaban para comunicarse por medio de llamadas de voz, sin embargo, en los años 90's fueron creados los SMS (Short Message Service). (Cárdena G 2012)

Podemos decir que el inventor de los SMS es Matti Makkonen, pero no recibió muchos créditos por hacerlo ya que no patentó su invento. El formato de 160 caracteres en los SMS fue determinado un año después por Friedham Hillebrand. Esto lo hizo porque se dio cuenta que 160 caracteres son suficientes para escribir un enunciado promedio.

El primer SMS que se mandó fue en 1992 por Brit Neil Papworth. Su primer texto fue "Happy Christmas" (Feliz Navidad), este mensaje fue enviado al director de Vodafone Richard Jarvis. El mensaje se mando desde una computadora hasta un Orbitel 901 Handset. (Cárdena G 2012) 
Después de haber sido creados los SMS, tomó 7 años para que los usuarios pudieran enviar SMS a usuarios utilizando el mismo operador. Fue hasta 1999 que los mensajes de texto se pudieron enviar entre diferentes redes y operadores. (Cárdena G 2012)

Actualmente, el servicio de SMS es de los más usados en los dispositivos móviles, con un 74\% de usuarios que se comunican por este medio en todo el mundo. (Cárdena G 2012)

Funcionalidad e importancia de los dispositivos móviles

Hoy en el mundo de la tecnología tenemos diferentes artefactos alrededor del mundo que forman parte de nuestro día a día, estos son llamados "Dispositivos móviles".

Un dispositivo móvil, lo podemos definir, como un aparato de pequeño tamaño, el cual posee un sin fin de funciones, entre las cuales podemos mencionar, el procesamiento e intercambio de información, la conexión a alguna red, todo esto a través de una memoria interna e ilimitada.

Entre los dispositivos móviles más utilizados en la actualidad tenemos los siguientes:

- Teléfonos inteligentes: Ligeros, portables, diversas aplicaciones y funciones entre la más destacadas e importantes tenemos, recibir y realizar llamadas telefónicas, así como también, captura de fotos con la cámara y vídeos

- Tablet's: No es diferentes a los teléfonos inteligentes, ya que en ellos poseemos también una gran variedad de aplicaciones, como agenda, organizadores, video llamadas y lectura de libros digitalmente 
- Cámaras digitales: Captura de momentos especiales, a través de fotografías o vídeos con un gran almacenamiento y una memoria expandible

- Videoconsola portátil: Básicamente su funcionamiento se basa en el entretenimiento portátil, con navegación a Internet y almacenamiento de archivo.

- Reproductores de música: Llevar la música que quieras a donde vayas en un solo dispositivo.

- Laptop: Almacenamiento de archivos de forma cómoda y potable, en lugar del uso de una PC.

La importancia de estos dispositivos móviles en la actualidad va de la mano con su dependencia generada por aquellas personas que hacen del dispositivo móvil parte de su vida, usándolo una gran cantidad de tiempo en el transcurso de su día, bien sea por motivos de trabajos o simplemente por entretenimiento u ocio. (Murgueytio L . 2014)

\section{Seguridad de los dispositivos móviles}

Hoy en día prácticamente todo llevamos en nuestro bolsillo un terminal móvil de última generación. Los smartphones han pasado a formar parte de nuestras vidas y un objeto imprescindible en nuestro día a día. En estos teléfonos, llevamos una ingente cantidad de información así como datos muy preciados que forman parte del valor intangible de nuestras empresas y que por tanto debemos proteger adecuadamente. Contraseñas, contactos, fotos, vídeos, correos electrónicos... o hasta datos de nuestras tarjetas de crédito es algo habitual en nuestros terminales. ¿ qué hago entonces si pierdo mi teléfono o me lo roban? Pues esta debería ser una cuestión que nos planteemos todos seriamente antes de tener que afrontar una situación real en un momento de crisis. 


\section{Medidas de seguridad básicas}

- Proteger nuestro móvil con una contraseña o patrón: Parece algo elemental y lo es pero aún se puede ver que mucha gente no lo hace. En caso de pérdida o robo lo único que perderíamos sería el hardware pero la persona que se haga con el dispositivo no debería poder acceder a la información que contiene ni poder suplantar nuestra identidad accediendo a correos electrónicos, herramientas de mensajería o aplicaciones de banca online por poner algunos ejemplos.

- Apuntar el IMEI para poder anular el dispositivo: El IMEI se obtiene pulsando *\#06\# deberíamos apuntar este código pues en caso de robo o extravío podremos llamar a nuestra operadora e inutilizar el terminal, una vez más conseguimos que no se acceda a la información.

- Tener instaladas aplicaciones de control remoto del dispositivo: Habitualmente en las versiones más actuales se incorporan de serie estas herramientas pero sino se pueden descargar de las respectivas tiendas de aplicaciones. Consisten en geolocalizar el dispositivo desde una cuenta vinculada y saber en el momento en que haya desaparecido donde se encuentra.

- Realizar copias de seguridad de la información del dispositivo: Hoy en día existen numerosas herramientas de respaldo en la nube de la información que contienen los dispositivos móviles. Si perdemos uno de ellos el disgusto debe ser la pérdida del hardware pero jamás de la información que se puede y debe poder restaurar de manera instantánea en otro dispositivo sin problema alguno. Por simplificarlo todo se ha de acabar aquella frase de 
"he perdido el móvil y ya no tengo contactos" esto (y el resto de información importante con mayor motivo) debería estar respaldada en una herramienta de Internet.

Además de estas medidas básicas que todos deberíamos implantar por seguridad no solo en dispositivos empresariales sino también personales existen otra serie de potenciales peligros que debemos conocer y tomar medidas:

\section{Otras medidas a tener en cuenta y riesgos a conocer:}

- Instalar un antivirus

- Cuidado con las redes públicas.

- Los sms, la mensajería instantánea y el aumento de las estafas

- Evitar hacer rooting o jailbreaking de los dispositivos (Seguridad en los dispositivos móviles. Smartphones y Tablets.2015).

Tendencia actual de los dispositivos móviles en la educación

La sociedad en que vivimos ha sido definida como la sociedad del conocimiento/información, es altamente competitiva, dinámica, variable y compleja (Castro y González-Palta, 2016). Se caracteriza, entre otros aspectos, por el rápido incremento en la producción de conocimiento, los procesos de innovación, el uso creciente y sistemático de nuevas tecnologías. Esta celeridad también es observable en el mundo laboral y académico-estudiantil, que, en continuo cambio, demanda a las universidades la formación de profesionales autónomos, que actualicen permanentemente sus conocimientos y competencias para hacer frente a las necesidades, también dinámicas, de la sociedad (Castro y González-Palta, 2016). La evolución de las Tecnologías de Información y Comunicación (TIC) ha permitido generar nuevos escenarios educativos para 
propiciar el aprendizaje y favorecer con ello el desarrollo de modalidades educativas con una mejor adaptación a las necesidades de los estudiantes. (Basantes, A V et al 2017)

En el ámbito educativo las TIC provee un sin número de herramientas, recursos, medios y formatos que posibilitan estrategias didácticas para facilitar la construcción de conocimientos, estos son: aulas virtuales, blogs didácticos, evaluaciones online, aprendizaje móvil, realidad virtual, entornos virtuales $3 \mathrm{~d}$, entre otros.

No obstante, su éxito depende de la capacidad para integrar la tecnología en el plan de estudios y crear experiencias de aprendizaje personalizado para cada alumno transformando el aula en un entorno de aprendizaje colaborativo (Briede, et al., 2015).

Según Basantes, A V (et al 2017 una de las variantes más exitosas hasta el momento es el Aprendizaje Móvil o m-learning, que se interpreta, como una oportunidad más para seguir aprendiendo con dispositivos móviles, generalmente en un marco de referencia educativo diferente al de un salón de clase y un profesor . La motivación para aprender juega un rol fundamental en cualquier campo de estudio; y el uso de los dispositivos móviles, bien concebido es una contribución importante a la motivación por el aprendizaje en los nuevos tiempos.

La revolución tecnológica que envuelve al mundo ejerce una influencia muy amplia en la manera de vivir de los seres humanos contemporáneos, lo que también ha impactado en la manera de enseñar y aprender. En el 2013, el 72\% de niños menores de ocho años ha utilizado un dispositivo móvil para algún tipo de actividad, ya sea en forma lúdica, o para videos. En comparación con el 2011 el incremento fue de un 34\%, únicamente el 38\% usó los dispositivos en el año referido. (Basantes, A V et al 2017) 
Sobre la base de las consideraciones anteriores se realizó una investigación en la Facultad de Educación Ciencia y Tecnología, de la Universidad Técnica del Norte. Ecuador con la finalidad de determinar la utilización de los dispositivos móviles en el proceso de aprendizaje. Se evidenció que los dispositivos móviles son subutilizados en el proceso de enseñanza-aprendizaje. (Basantes, A V et al 2017).

Según se ha visto durante los próximos quince años, la puesta en marcha de proyectos de aprendizaje móvil y los modelos pedagógicos que éstos adopten se deberían guiar no sólo por las ventajas y las limitaciones de las tecnologías móviles, sino también por el análisis de cómo encajan esas tecnologías en el tejido social y cultural de las comunidades. La educación y la tecnología pueden y deben evolucionar en paralelo y apoyarse mutuamente. (Shuler C , 2013)

Existe una experiencia de uso de estos dispositivos en el aula de Matemática Financiera, para realizar diversos cálculos correspondientes a las diferentes operaciones de inversión y financiamiento, objeto de estudio de la asignatura, mediante el uso de aplicaciones que permiten operar con calculadoras financieras y planillas de cálculo de uso libre, nos permite comprobar que los jóvenes aprenden mejor y están más predispuestos a la incorporación de nuevos contenidos, cuando encuentran una conexión con el entorno, desarrollando un interés personal hacia lo nuevo.( Bravino L, Margaría O . 2014)

\section{Ventajas y Desventajas de los dispositivos móviles como herramienta educativa}

\section{Principales ventajas:}


Movilidad. Livianos y trasportables, pueden ser utilizados tanto dentro como fuera del aula, permitiendo la búsqueda, selección y procesamiento de la información, la colaboración y la coconstrucción del conocimiento en horario escolar y/o extraescolar.

Conectividad. La conexión inalámbrica facilita; sin la mediación de cableado, la unión de dos o más dispositivos, el acceso y uso de internet, intercambio de información y trabajo colaborativo. Favorece la comunicación, la creación de redes y las comunidades vituales de aprendizaje.

La llamada red mesh por ejemplo, permite que cada dispositivo se transforme en un nodo y cada nodo establezca una comunicación con los demás nodos y con la red.

Funcionalidad. Estos dispositivos cuentan con una batería y sólo el acceso a la red está limitado por la existencia en el lugar de red inalámbrica. La capacidad de procesamiento de datos facilita la recopilación de la información en cualquier contexto.

\section{Principales desventajas}

Precio. Si bien se trata de dispositivos más baratos que una computadora fija de mesa, en algunos casos -hablamos de los PDA, los TabletPC, etc. no de las laptops que rondan en el mercado uruguayo en alrededor de 1000 dólares americanos- su precio no es accesible a la toda de la población, lo que no favorecería la generalización a nivel escolar sin la intervención de políticas públicas.

Tamaño. Según el dispositivo, su teclado y su pantalla pueden ser de mayor o menor tamaño. 
Esto hace más incómodo su uso, en espacial si hablamos de estudiantes, no acostumbrados a la tecnología moderna -por ejemplo teléfonos móviles- para los niños incluso, "nativos digitales", requiere de una adaptación.

En cuanto a la tecnología de difusión de la información -no para las laptops pero sí para otros dispositivos más pequeños- la misma debe estar adaptada para que se pueda acceder desde el mismo -tamaño de pantalla, puede incluso, no visualizar la misma difundida a partir de determinado software.

Funcionalidad. Por más que la industria está realizando grandes esfuerzos para dotarlos de fortaleza física, los mismos siguen teniendo fragilidad y algunos pueden ser fáciles de extraviar. Es preciso instruir y responsabilizar a los alumnos y las familias en el uso responsable y cuidado personal de los mismos. (Rabajoli G 2007)

\section{Aplicación de la tecnología móvil en las empresas}

En la actualidad, uno de los aspectos vitales y en crecimiento de los negocios está constituido por las Tecnologías de la Información (TI), las cuales apoyan a todo tipo de organización mejorando su eficacia y eficiencia tanto en los procesos propios de la actividad como en la toma de decisiones gerencial y la colaboración entre grupos de trabajo sin importar su situación geográfica. De esta manera se fortalece la competitividad de la empresa, permitiéndole afrontar el cambiante mercado globalizado. (Carrasco Usanos S. 2015)

En el periodo económico actual, protagonizado por los emprendedores, son muchos los que ven nuevas oportunidades en las aplicaciones móviles. Éstas impulsan la innovación y originan nuevos negocios. (Carrasco Usanos S. 2015) 
Los dispositivos móviles hacen que la información importante de la empresa pueda estar disponible en todo momento, acelerando la toma de decisiones y aumentando la productividad. Con las políticas adecuadas, las organizaciones se beneficiarán de la flexibilidad y eficiencia que esto supone. (Carrasco Usanos S. 2015)

Así mismo, los potenciales clientes utilizan cada vez más sus dispositivos móviles con acceso a Internet para navegar por las páginas web de las empresas e incluso realizar compras, es por ello que las estrategias de las empresas han de procurar que la experiencia de estos usuarios sea satisfactoria. (Carrasco Usanos S. 2015)

Es evidente que adoptar estas iniciativas de movilidad conllevará beneficios para las empresas, sin embargo, hay que tener en cuenta los posibles riesgos a los que se pueden enfrentar como, por ejemplo, el robo o pérdida de dispositivos móviles o el acceso no autorizado. Es muy importante no olvidarse de implementar estrategias de seguridad. (Carrasco Usanos S. 2015).

Por último, el éxito de la implantación de un sistema de información que esté basado en la tecnología móvil, no se debería medir únicamente por su eficiencia en la reducción de costes y tiempos; sino también por su eficacia a la hora de apoyar las estrategias de una empresa u organización, al mejorar la estructura y cultura organizacional y al otorgar un valor añadido a los clientes, proveedores u otras partes interesadas.( Carrasco Usanos S. 2015)

A manera de colofón el mundo en que vivimos nos ofrece hoy múltiples herramientas y dispositivos que nos permiten realizar innumerables tareas vinculadas al ocio, a la comunicación, al desempeño laboral, a la información, turismo, sanidad a la educación, entre otras 


\section{Bibliografía.}

BASANTES, A V.; NARANJO, M E.; GALlEGOS, M C.; NHORA M., B Los Dispositivos Móviles en el Proceso de Aprendizaje de la Facultad de Educación Ciencia y Tecnología de la Universidad Técnica del Norte de Ecuador Formación Universitaria, vol. 10, núm. 2, 2017, pp. 7987

BRIEDE, J.C., LEAL, I.M., MORA, M. L., Y PLEGUEZUELOS, C.S., 2015Propuesta de Modelo para el Proceso de Enseñanza Aprendizaje Colaborativo de la Observación en Diseño, Utilizando la Pizarra Digital Interactiva (PDI), Formación Universitaria.

BRAVINO L , MARGARÍA O . 2014. Dispositivos móviles: una experiencia en el aula de Matemática Financiera. [consulta 25 sep 2017]. Disponible en: www.oei.es/historico/congreso2014/memoriactei/840.pdf

CARDENA G 2012. Historia de los dispositivos móviles. [consulta 25 sep 2017]. Disponible en: http://dispmovs.blogspot.com/2012/03/historia-de-los-dispositivos-moviles.html

CASTRO, P.J., Y GONZÁLEZ-PALTA, I.N., 2016 Percepción de Estudiantes de Psicología sobre el Uso de Facebook para Desarrollar Pensamiento Crítico: Revista Formación Universitaria, doi: 10.4067/S0718- 50062016000100006, (en línea), 9 (1), 45-56

CARRASCO USANOS S. 2015. Análisis de la aplicación de la tecnología móvil en las empresas. TRABAJO FINAL DE CARRERA Licenciatura en Administración y Dirección de Empresas. [consulta 25 sep 2017]. Disponible en :https://riunet.upv.es/bitstream/handle/10251/.../TFC\%20Silvia\%20Carrasco.pdf?

Historia del móvil [sitio web]. 2013. [consulta 25 sep 2017]. Disponible en https://cricari.wordpress.com/

MURGUEYTIO L. 2014. Funcionalidad e importancia de los dispositivos móviles [consulta 25 sep 2017]. Disponible en https://sites.google.com/site/appsdispositivosymas/contenido/pagina-cuatro

RABAJOLI G 2007. Ventajas y Desventajas de los dispositivos móviles. [consulta 25 sep 2017]. Disponible en http://pidoayuda.blogspot.com/2007/09/ventajas-y-desventajas-de-los.html

RODRÍGUEZ LUIS F. 2012. Telecomunicaciones: Historia y Conceptos Básicos. [consulta 25 sep 2017]. Disponible en ftp://ftp.crya.unam.mx/pub/luisfr/suprema/Telecomunicaciones.doc

Seguridad en los dispositivos móviles. Smartphones y Tablets [sitio web]..2015. [consulta 25 sep 2017]. Disponible en http://www.fundacionctic.org/sat/articulo-seguridad-en-los-dispositivos$\underline{\text { moviles-smartphones-y-tablets }}$

SHULER C , WINTERS N , WEST M 2013. El future del aprendizaje movil . UNESCO. [consulta 25 sep 2017]. Disponible en unesdoc.unesco.org/images/0021/002196/219637s.pdf 